Conclusion This result shown that the most contamination in NICU is from gram negative bacteria.

\section{P0-0561 THE IMPACT OF THE SEPSIS SEVERITY TO THE OXYGENATION OF THE IMMATURE NEONATAL BRAIN}

${ }^{1} \mathrm{D}$ Rallis, ${ }^{2} \mathrm{P}$ Karagianni, ${ }^{2} \mathrm{E}$ Mylona, ${ }^{2} \mathrm{~N}$ Nikolaidis, ${ }^{2} \mathrm{C}$ Tsakalidis. ${ }^{1}$ Paediatrics, Aghia Sophia Children's Hospital, Athens, Greece; 'Neonatal Unit, Papageorgiou General Hospital, Thessaloniki, Greece

\subsection{6/archdischild-2014-307384.1203}

Background and aims Sepsis is major cause of neonatal morbidity and mortality. The level of organ dysfunction is related to the severity of sepsis, as milder episodes seem to lead to less injury. Our aim was to evaluate differences in brain oxygenation, measured by Near Infrared Spectroscopy (NIRS) and estimated via TOI (Total Oxygen Index) and FTOE (Fraction Tissue Oxygen Extraction), according to the sepsis severity.

Methods We designed a prospective study in the 2nd NICU of AUTH, Greece, between 6/2012-12/2012. Neonates with confirmed sepsis underwent 3 NIRS measurements on day 1, 3 and 7 of the episode. Sepsis was classified according to IPSCC criteria into: Sepsis, Severe Sepsis and Septic Shock.

Results Fifty neonates were enrolled with equal birthweight (sepsis: 1610 gr, severe sepsis: 1670 gr, septic shock: 1550 gr) and gestational age $(31,31,30$ weeks respectively).

A TOI decrease/FTOE increase in the 7 th day was recorded in all groups (Sepsis: TOI: 68, 70, 62, FTOE: 29\%, 27\%, 35\%, Severe sepsis: TOI: 66, 70, 62, FTOE: 29\%, 27\%, 33\%, Septic Shock: TOI: 64, 66, 61, FTOE: 33\%, 29\%, 35\%). Neonates with severe sepsis and septic shock required more intensive management and had worst outcome. The brain oxygenation, however, was equally decreased in all 3 groups $(\mathrm{p}>0.05)$, irrespectively to the severity of the septic episode.

Conclusion The outcome of the neonates is proportionally depended to the severity of sepsis, however, even the milder forms seem to cause significant decrease on the brain oxygenation and potential equal brain injury.

\section{P0-0562 PREVALENCE OF GRAM NEGATIVE ORGANISMS ON ROUTINE SURVEILLANCE IN A TERTIARY NEONATAL INTENSIVE CARE UNIT}

${ }^{1}$ MJ Cawsey, 'SV Rasiah, ${ }^{2} \mathrm{~J}$ Gray. 'Neonatal Intensive Care Unit, Birmingham Women's NHS Foundation Trust, Birmingham, UK; ${ }^{2}$ Microbiology, Birmingham Women's NHS Foundation Trust, Birmingham, UK

\subsection{6/archdischild-2014-307384.1204}

Background and aim We perform weekly surveillance for gram negative organisms by rectal swabs and surface swabs for all babies admitted to the neonatal unit. The aim of the audit was to look at the prevalence of colonisation with gram negative bacteria (GNB) and the outcome of these babies.

Methods This was a retrospective review of all positive rectal and surface swabs from $01 / 04 / 13$ to $31 / 03 / 14$.

Results In the last year we had 1465 admissions. The results of the screening programme are shown in the table below. These included GNB on surface or rectal swabs.

Conclusion Preterm babies are at an increased risk of being colonised with GNB at a later date whilst on the neonatal unit. In comparison, term babies were likely to have incidental GNB isolates earlier on. Having a surgical procedure increased the

\begin{tabular}{lll} 
Abstract P0-0562 Table 1 & & \\
\hline & Preterm & Term $>37$ weeks \\
Admissions $n$ & 705 & 760 \\
$n$ with positive swab & $65(9.2 \%)$ & $13(1.7 \%)$ \\
Median day of isolation & $14(0-115)$ & $3(1-40)$ \\
& $28^{+3}$ & $39^{+6}$ \\
Median gest age & $\left(23^{+5}-36^{+5}\right)$ & $\left(37^{+1}-42^{+0}\right)$ \\
& 1060 & 3180 \\
Median birth weight & $(500-3260 \mathrm{~g})$ & $(2280-4820 \mathrm{~g})$ \\
Inborn:Outborn & $57: 8$ & $10: 1$ \\
$n$ had Surgery & $10(15 \%)$ & $2(15 \%)$ \\
Use of Meropenem & $28(43 \%)$ & $1(8 \%)$ \\
Organisms & & \\
Pseudomonas & 13 & 4 \\
ESBL & 10 & 4 \\
Serratia & 17 & 1 \\
Gent resistant organism & 18 & 2 \\
Acinetobacter & 7 & 2 \\
Outcome & & \\
Discharged home & 43 & 9 \\
Transferred out & 15 & 3 \\
Died & 5 & 0 \\
Inpatient & 2 & 1 \\
\hline
\end{tabular}

chances of being colonised with GNB. The use of Meropenem was increased in the preterm population. We routinely isolated these babies with GNB until discharge from the neonatal unit.

\section{PO-0563 DOES EARLY CRP PREDICT CHORIOAMNIONITIS IN VERY LOW BIRTH WEIGHT PRETERM INFANTS?}

${ }^{1}$ E Ryan, ${ }^{2} D$ Eves, ${ }^{2} \mathrm{P}$ Jayadev Menon, ${ }^{2} \mathrm{~S}$ Alnafisee, ${ }^{3} \mathrm{E}$ Mooney, ${ }^{3} \mathrm{P}$ Downey, ${ }^{1} \mathrm{EJ}$ Molloy. ${ }^{1}$ Neonatology, National Maternity Hospital, Dublin, Ireland; ${ }^{2}$ Paediatrics, Royal College of Surgeons, Dublin, Ireland; ${ }^{3}$ Pathology, National Maternity Hospital, Dublin, Ireland

\subsection{6/archdischild-2014-307384.1205}

Background Chorioamnionitis (CA) is associated with preterm birth and adverse neonatal outcomes. The correlation between stage of histological CA, and haematological parameters in the early postnatal period are incompletely defined.

Aim To examine the relationship between CRP and grade of CA in VLBW infants and any associations with neonatal outcomes.

Methods A retrospective review of consecutive infants born $<32$ weeks gestation or $<1.5 \mathrm{~kg}$ at a single centre tertiary referral centre. CA on placental histology was reported as solely maternal inflammatory response (MIR) or both MIR and fetal inflammatory response (FIR). Demographics, haematological parameters and outcomes were recorded.

Results 509 infants were included and histological CA was found in $20 \%$ of placentas but $47.8 \%$ at $<28$ wks. CRP was raised above baseline $(>0.3 \mathrm{mg} / \mathrm{L})$ in $61.8 \%$ of infants with FIR, $64.7 \%$ of infants with MIR and $18.8 \%$ with no CA. CRP on day $1>5 \mathrm{mg} / \mathrm{L}$ was specific for $90.9 \%$ of FIR, 3\% of MIR, and $6.1 \%$ of infants no CA. CRP $>10 \mathrm{mg} / \mathrm{L}$ was $100 \%$ specific for CA but not sensitive.. Advanced FIR on histology (funisitis) correlated significantly with higher CRP. A pathogen was isolated in $0.6 \%$ of CA and blood cultures were positive in $0.02 \%$ of preterm infant with the following outcomes: died $(n=6)$; FIR ( $=8)$; Funisitis $(\mathrm{n}=5)$; High CRP $(\mathrm{n}=5)$. 